\title{
Análise sobre variáveis explicativas da autoeficácia docente ${ }^{1}$
}

\section{Analysis of explanatory variables of teacher self-efficacy}

\author{
Daniela Couto Guerreiro Casanova ${ }^{2}$ \\ Roberta Gurgel Azzi
}

\begin{abstract}
RESUMO
Esta pesquisa teve como objetivo analisar as possíveis relações explicativas entre variáveis pessoais, variáveis de atividade docente e variáveis contextuais e as crenças de autoeficácia docente de professores do ensino médio público paulista. Tal crença é entendida como o julgamento que o professor faz acerca de suas próprias capacidades para atingir resultados desejados de engajamento e aprendizagem dos alunos, mesmo entre aqueles que podem ser difíceis ou desmotivados, e tem sido associada à satisfação docente e ao sucesso escolar, dentre outras contribuições. Por meio de questionários obteve-se a participação de 201 educadores oriundos de 11 escolas do ABC Paulista, dos quais 180 eram professores do ensino médio e 21 eram gestores escolares. A análise de regressão linear multivariada constatou que as variáveis formação, crença de eficácia coletiva escolar e tempo de atuação na unidade escolar pesquisada mostraram-se significativamente explicativas da autoeficácia docente. A necessidade de se proporcionar formação docente que alie o desenvolvimento de habilidades e de autoeficácia, bem como condições para diminuir a rotatividade docente e fortalecer a crença de eficácia coletiva escolar são discutidas sob a ótica da teoria social cognitiva.

Palavras-chave: formação; eficácia coletiva; condições de trabalho; satisfação docente.
\end{abstract}

DOI: $10.1590 / 0104-4060.43236$

1 Agradece-se o apoio da Fundação de Amparo à Pesquisa do Estado de São Paulo (processo $\left.\mathrm{n}^{\circ} 2010 / 51657-1\right)$.

2 Universidade Estadual de Campinas. Faculdade de Educação. Campinas, São Paulo, Brasil. Av. Bertrand Russell, nº 801. Cidade Universitária Zeferino Vaz. CEP: 13083-865. E-mails: danielaguerreiro@yahoo.com.br; azzi@unicamp.br 


\begin{abstract}
This research aims to analyze the possible explanatory relationships among personal variables, teaching activities variables, contextual variables and the São Paulo state public high school teachers' self-efficacy beliefs. This thought is defined as teachers' perceptions about their own capabilities to achieve desired results of engagement and student learning, even among those cases that may be difficult or unmotivated, and it has been associated to teachers' satisfaction and to the school success, besides other contributions. By using questionnaires, 201 educators from 11 ABC Paulista schools participated, from which 180 were high school teachers and 21 were school managers. The analyzis of multivariate linear regression verified that the variables graduation, school collective efficacy belief and time of work at the same school were significant in order to explain the teachers' self-efficacy. Under the social cognitive theory the necessity of teacher graduation that allies ability and self-efficacy development, as well as conditions to minimize teachers' rotation and make the school collective efficacy belief stronger are discussed.
\end{abstract}

Keywords: graduation; collective efficacy; work conditions; teachers' satisfaction.

A crença de autoeficácia docente tem sido associada como um dos aspectos que contribuem para o estabelecimento de ambientes possibilitadores para o aprender dos estudantes. Essa crença, definida como "um julgamento que o professor faz acerca de suas próprias capacidades para atingir resultados desejados de engajamento e aprendizagem dos alunos, mesmo entre aqueles que podem ser difíceis ou desmotivados". (TSCHANNEN-MORAN; WOOLFOLK-HOY, 2001, p. 783), atua como mediadora de ações realizadas pelos professores, como a utilização de novas estratégias metodológicas e de ensino, e da reflexão docente sobre o objetivo estabelecido e a realização, ou não, desse objetivo. (THOONEN et al., 2011).

A motivação intrínseca e o engajamento nas atividades de ensino são comumente associadas a professores que se percebem autoeficazes. (RODRIGUEZ et al., 2009; THOONEN et al., 2011). Tais professores tendem a se identificar com a profissão, a sentirem maior satisfação com o trabalho docente, a se sentirem bem e entusiasmados com a atuação, aspecto que evidencia a autoeficácia como preventiva ao burnout docente. (CASTELO; LUNA, 2012; DUFFY; LENT, 2009; FERREIRA, 2011; SALANOVA; LLORENS, 2011; SKAALVIK; SKAALVIK, 2007).

As concepções que os docentes têm sobre a capacidade dos estudantes para aprender também são mediadas pela crença de autoeficácia docente. Professores que se percebem autoeficazes utilizam da persuasão para promover o 
interesse dos estudantes para o aprender, estimulando a aprendizagem autorregulada (BANDURA, 1995, 1997), entendendo os erros e as dificuldades de aprendizagem como parte do aprender, de modo que se assumem responsáveis pela promoção do aprender dos estudantes. Administram os conflitos e atuam de modo humanístico, não autoritário, proporcionando o desenvolvimento autônomo dos estudantes, aspectos que estimulam a motivação, a autoeficácia e o desempenho dos educandos. (AZZI; POLYDORO; BZUNECK, 2006; AZZI; POLYDORO, 2010; BANDURA, 1997; IAOCHITE, 2007; TSCHANNEN-MORAN; WOOLFOLK-HOY, 2001; WOOLFOLK-HOY; DAVIS, 2006).

A experiência direta de situações interpretadas como exitosas parece ser a fonte de maior contribuição para a percepção de autoeficácia docente. (CASTELO; LUNA, 2012). Já a persuasão social advinda pelo reconhecimento dos estudantes e a percepção de apoio ao docente por parte dos gestores também se relacionam ao julgamento de autoeficácia docente (CASTELO; LUNA, 2012; IAOCHITE, 2007; FERREIRA, 2011; MACEDO, 2009), principalmente para os docentes menos experientes. (IAOCHITE, 2007).

A literatura analisada, entre outros objetivos, tem se dedicado à busca de compreensão sobre a contribuição de algumas variáveis pessoais, de atividade docente e de contexto para a construção da crença de autoeficácia docente. Dentre as variáveis pessoais, identificou-se que os homens parecem se perceber mais capazes para utilizar tecnologias como estratégia de ensino (ALVARENGA, 2011), manter a disciplina, cooperar com os colegas de profissão e com os pais dos estudantes e as mulheres demonstraram ter percepções de autoeficácia docente mais altas do que os homens para ensinar literatura inglesa. (TSCHANNEN-MORAN; JOHNSON, 2011).Os professores mais velhos demonstraram maiores percepções de autoeficácia docente quando considerados os professores de escolas públicas do Estado do Paraná (MACEDO, 2009) e do Estado de Minas Gerais. (FERREIRA, 2011).

Quanto às variáveis de atividade docente, o tempo de experiência docente parece relacionar-se à percepção de autoeficácia docente quando considerados os professores de escolas públicas do Estado de Minas Gerais (FERREIRA, 2011) e do Estado do Paraná (MACEDO, 2009), sendo que os professores em início de carreira parecem apresentar crenças de autoeficácia docente mais frágeis, de modo que seria interessante evitar que os professores em início de carreira lidassem com as classes entendidas como desafiadoras. (TSCHANNEN-MORAN; HOY, 2001). Professores com titulação em nível de Pós-Graduação demonstraram percepções de autoeficácia docente mais fortes do que os professores licenciados em contexto norte-americano. (TSCHANNEN-MORAN; JOHNSON, 2011), como também no contexto brasileiro, especificamente para a autoeficácia computacional (ALVARENGA, 2011), sendo que professores que se 
percebem mais bem preparados também se percebem mais autoeficazes. (FERREIRA, 2011; IAOCHITE, 2007; TSCHANNEN-MORAN; JOHNSON, 2011).

Professores com maiores cargas de jornada de trabalho se julgaram mais autoeficazes do que professores com jornadas de trabalho de menor duração. (IAOCHITE, 2007; OLIVEIRA; FREITAS, 2010). A não concordância dos professores com as novas organizações do trabalho docente podem relacionar-se negativamente com a percepção de autoeficácia docente. E, quanto maior a percepção de liberdade de expressão, de autonomia docente para conduzir as próprias aulas e para influenciar as decisões pedagógicas, maior a percepção de autoeficácia docente. (SKAALVIK; SKAALVIK, 2007).

As variáveis de contexto com as quais os docentes têm de lidar cotidianamente também se relacionam com a percepção de autoeficácia docente. Professores de escolas privadas tendem a ter percepções de autoeficácia pouco mais altas do que os atuantes em escolas públicas. (CASTELO; LUNA, 2012; FREITAS; OLIVEIRA, 2010). A maioria dos professores que atua no ensino fundamental demonstrou percepções de autoeficácia mais fortes do que os professores que atuam no ensino médio e na educação infantil. (FREITAS; OLIVEIRA, 2010). A autoeficácia docente parece não se diferenciar de acordo com a localização das escolas (GOYA; BZUNECK; GUIMARÃES, 2008), mas com a adequada infraestrutura da escola e o tamanho das turmas sim, de modo que escolas com melhores infraestruturas e turmas menores estão associadas a professores com maiores percepções de autoeficácia docente. (IAOCHITE, 2007; TSCHANNEN-MORAN; JOHNSON, 2011).

Como pode ser observado nesta breve introdução, a autoeficácia docente pode auxiliar na realização de práticas escolares que possam contribuir para transformar o aprender dos estudantes, desde que asseguradas condições de trabalho adequadas. Para tanto, coloca-se como aspecto fundamental que tal crença seja mais bem compreendida, de modo que se possa desenvolver ações, de formação ou de intervenção escolar, visando auxiliar no fortalecimento dessa crença. (AZZI; POLYDORO, 2010; BANDURA, 1995). Diante disso, esta pesquisa tem como objetivo analisar as possíveis relações explicativas entre variáveis pessoais, variáveis de atividade docente e variáveis contextuais e as crenças de autoeficácia docente de professores do ensino médio público paulista.

\section{Método}

Esta pesquisa foi realizada após a autorização da Comissão de Ética em Pesquisa da Faculdade de Ciências Médicas da Unicamp (protocolo nº 997/2010, 
processo CAAE 0777.0.146.000-10). Seguindo orientação da Diretoria de Ensino de São Bernardo do Campo, em cada unidade escolar, a pesquisa só foi realizada após autorização da diretora responsável, mediante assinatura da Carta de Autorização Escolar.

\section{Procedimentos de coleta de dados}

Considerando-se a localização das escolas, 25 escolas foram contatadas por meio de ligação telefônica, das quais 12 escolas se disponibilizaram a marcar uma reunião com a pesquisadora e obter explicação dos objetivos, dos procedimentos e da voluntariedade da participação na pesquisa. Onze diretores autorizaram a realização da pesquisa na escola em que atuavam e agendaram os dias em que as coletas seriam realizadas, sempre durante reuniões de Atividade de Trabalho Pedagógico Coletivo (ATPC), com a participação dos coordenadores, professores e da pesquisadora. Para responder os questionários, os participantes utilizaram, em média, 50 minutos. A pesquisadora restringiu-se a dar as informações necessárias para o correto preenchimento dos instrumentos e a solicitar que os participantes não trocassem informações sobre os instrumentos utilizados durante o momento de sua aplicação, a fim de se garantir a confiabilidade das respostas. Além disso, os participantes receberam a garantia do sigilo das respostas, bem como a garantia de que as respostas seriam utilizadas exclusivamente para fim de pesquisa.

\section{Materiais de coletas de dados}

Para elucidar a finalidade dos materiais utilizados, suas características serão descritas:

Questionário de Caracterização dos Participantes: Trata-se de um questionário com perguntas objetivas em relação a: gênero, idade, tempo de exercício do magistério, tempo de atuação na escola pesquisada, tipo de formação profissional, tipo de vínculo empregatício, tempo dedicado à jornada de trabalho, quantidade de alunos por turma, percepção sobre a infraestrutura da escola, entre outras características pessoais e profissionais.

$>$ Escala de Autoeficácia Docente - versão curta (CASANOVA, 2013; TSCHANNEN-MORAN; WOOLFOLK-HOY, 2001): essa escala dedica-se 
a obter a percepção sobre a autoeficácia docente. É composta por 12 itens, em formato Likert de 10 pontos, organizados em três dimensões: eficácia nas estratégias instrucionais (variância explicada $=0,51 \% ; \alpha=0,81$ ), eficácia no manejo de sala de aula (variância explicada $=0,62 \% ; \alpha=0,81$ ) e eficácia no engajamento do estudante (variância explicada $=0,69 \% ; \alpha=0,80$ ). O MSA de Kaiser $(0,90)$ e a consistência interna da escala como um todo $(\alpha=0,91)$ contribuem para confirmar as evidências de validade adequadas a essa escala.

$>$ Questionário do Gestor (CASANOVA, 2013; TSCHANNEN-MORAN; GAREIS, 2004): dedicado a obter a percepção de autoeficácia dos gestores escolares, essa escala é composta por 18 itens, em formato Likert de 10 pontos, com organização unifatorial. O MSA de Kaiser de 0,80 , a variância explicada de $73 \%$ e a consistência interna de 0,95 sugerem evidências de validade adequadas ao objetivo dessa escala.

> Escala de Eficácia Coletiva Escolar (CASANOVA, 2013; TSCHANNEN-MORAN; BARR, 2004): essa escala é destinada à obtenção da percepção da eficácia coletiva escolar, sendo composta por 12 itens, em formato Likert de 10 pontos, organizados em dois fatores: estratégias instrucionais (variância explicada de $65 \% ; \alpha=0,93$ ) e disciplina dos estudantes (variância explicada de $7 \% ; \alpha=0,91$ ). Com MSA de Kaiser de 0,94, variância explicada total de $72,6 \%$ e consistência interna total de 0,95 , essa escala mostrou-se adequada para o fim pretendido.

Índice Paulista de Vulnerabilidade Social (IPVS): esse índice, elaborado e disponibilizado pela Fundação Sistema Estadual de Análise de Dados (SEA$\mathrm{DE}$ ), divulga informações sobre as condições de vida e o bem-estar de pessoas, famílias e/ou comunidades do Estado de São Paulo. Tal índice classificou áreas geográficas de acordo com os recursos aos quais a população ali residente tinha acesso, sendo: (1) nenhuma vulnerabilidade; (2) vulnerabilidade muito baixa; (3)vulnerabilidade baixa; (4) vulnerabilidade média; (5) vulnerabilidade alta; e (6) vulnerabilidade muito alta (SEADE, 2012). Por meio de acesso ao site do SEADE foi possível obter o IPVS referente ao bairro em que se localiza cada escola aqui pesquisada.

\section{Participantes}

Foram considerados participantes 201 professores e gestores que estavam presentes nas coletas de dados e aceitaram participar da pesquisa mediante assinatura do Termo de Consentimento Livre e Esclarecido. Destes, 182 parti- 
cipantes eram oriundos de dez escolas localizadas em São Bernardo do Campo e 19 participantes de uma escola localizada em São Caetano do Sul. Quanto às funções, foram seis diretores, seis vice-diretores, nove coordenadores pedagógicos e 180 professores de ensino médio. Sobre os aspectos pessoais, pode-se dizer que 141 participantes eram do gênero feminino, 55 eram do gênero masculino e 5 não responderam essa questão; a idade média da amostra era de 43,56 anos $(\mathrm{DP}=9,85$, idade mínima $=23$ e idade máxima $=67$ anos $)$.

Quanto aos aspectos de atividade docente, 16 participantes eram graduados em pedagogia, 97 eram graduados em alguma licenciatura, 58 aprofundaram seus estudos em cursos de especialização, 14 eram mestres, 4 eram doutores e 12 participantes não responderam essa questão. Essa amostra tinha em média 15,77 anos de experiência docente e/ou na educação escolar ( $\mathrm{DP}=8,66$; mínimo $=0$ e máximo $=39) ; 13,68$ anos em média de atuação na função exercida na ocasião da coleta de dados $(\mathrm{DP}=8,61$, mínimo $=0$ e máximo $=37)$; e 5,94 anos de atuação na escola pesquisada $(\mathrm{DP}=5,65$, mínimo $=0$ e máximo $=35)$.

A carga horária da jornada de trabalho semanal era de 33,99 horas em média $(\mathrm{DP}=12,17$, mínimo $=6$ e máximo $=70)$. Essa jornada era cumprida apenas na escola pesquisada para a maioria da amostra $(n=129)$, alguns a cumpriam na escola pesquisada e em mais uma escola pública $(n=37)$, outros a cumpriam em mais uma escola privada $(\mathrm{n}=20)$, uma minoria cumpria essa jornada em mais uma escola pública e em mais uma escola privada $(n=6)$ e 9 participantes não responderam essa questão. Dos professores, 54 lecionavam apenas no ensino médio, 118 atuavam no ensino médio e no ensino fundamental - séries finais -, 2 atuavam no ensino médio e na educação de jovens e adultos (EJA) e 6 atuavam no ensino médio, no ensino fundamental - série finais - e na educação de jovens e adultos (EJA).

Quanto às turmas, 4 participantes trabalhavam com turmas compostas por 30 estudantes; 43 participantes lecionavam para turmas compostas por 35 estudantes; 91 participantes lecionavam para turmas com 40 estudantes; 49 participantes trabalhavam com turmas de 45 estudantes; 4 participantes lecionavam para turmas com 50 estudantes; e 11 participantes não responderam essa questão. A infraestrutura da escola foi considerada insuficiente por 30 participantes, pouco adequada por 60 participantes, adequada por 88 participantes e muito adequada por 16 participantes, sendo que 7 não responderam essa questão. De acordo com o IPVS as escolas participantes identificadas como 2, 11, 13 e 17 localizavam-se em área classificada como Grupo 1, nenhuma vulnerabilidade; as escolas identificadas como 3, 4, 12 e 15 localizavam-se em área classificada como Grupo 2, vulnerabilidade muito baixa; e as escolas identificadas como 1, 14 e 16 localizavam-se em áreas classificadas como Grupo 4, vulnerabilidade média. 


\section{Procedimentos de análise de dados}

As análises estatísticas foram executadas com a utilização do programa The Statistical Analysis System (SAS), versão 9.2. Os instrumentos respondidos foram submetidos à inspeção visual, excluindo-se os que não tiveram, pelo menos, $90 \%$ dos itens respondidos. A confiabilidade da digitação foi verificada em $20 \%$ dos casos escolhidos de forma aleatória, bem como por meio da análise das frequências das respostas.

Quanto aos casos válidos, para a análise descritiva (mediana, média, valores mínimos e máximos) foram utilizadas todas as respostas válidas e para as análises de regressão lineares univariadas e multivariadas foram utilizados apenas os participantes que responderam todos os itens das escalas analisadas, de modo que poderá ser verificada redução da amostra para as análises multivariadas. Por meio do teste Shapiro-Wilk verificou-se desvio significativo da normalidade $(\mathrm{p}<0,05)$, fato que levou à transformação dos dados em postos para viabilizar a análise de regressão multivariada de dados. (CONOVER; IMAN, 1981).

Os resultados verificados por meio da análise de regressão linear univariada serão expostos considerando-se: o $\mathrm{R}^{2}$, que se refere à correlação de uma variável explicativa com a variável critério e a porcentagem da variância da variável critério explicada pela variável explicativa; o peso padronizado $(\beta)$ das variáveis explicativas, que fornece informações sobre a importância de cada variável explicativa; o erro padrão (EP), que fornece uma medida sobre o quão correta está a estimativa da análise realizada; e o valor de p, que indica se a relação verificada é significativa ou não.

Os resultados verificados por meio da análise de regressão linear multivariada serão expostos considerando-se: o $\mathrm{R}^{2}$ total, que se refere à correlação entre todas as variáveis explicativas juntas e a variável critério, bem como a toda variância das variáveis explicativas em relação à variável critério; o $\mathrm{R}^{2}$ parcial, que se refere à correlação entre uma variável explicativa e a variável critério; o peso padronizado das variáveis explicativas $(\beta)$, que fornece informações sobre a importância de cada variável explicativa; o erro padrão (EP), que fornece uma medida sobre o quão correta está a estimativa da análise realizada; e o valor de $\mathrm{p}$, que indica se a relação verificada é significativa ou não. 


\section{Resultados}

Os participantes demonstraram percepções classificadas como "capaz" (entre 7 e 8 na escala de resposta), constatado pela mediana 7,50 (M=7,49; $\mathrm{DP}=1,01 ;$ mínimo $=4,17$ e máximo $=10 ; \mathrm{n}=159)$. Quanto aos fatores que compõem a autoeficácia docente, observou-se que a autoeficácia para estratégias instrucionais revelou uma mediana $(8,00)$ um pouco maior $(\mathrm{M}=8,10$; $\mathrm{DP}=$ 1,04; mínimo $=4$ e máximo $=10 ; \mathrm{n}=159)$ do que as dimensões autoeficácia para o manejo de sala de aula $(\mathrm{Md}=7,25 ; \mathrm{M}=7,30 ; \mathrm{DP}=1,13$; mínimo $=4 \mathrm{e}$ máximo10; $\mathrm{n}=159)$ e autoeficácia para o engajamento dos alunos $(\mathrm{Md}=7,00$; $\mathrm{M}=7,08 ; \mathrm{DP}=1,29 ;$ mínimo $=3$ e máximo 10; $\mathrm{n}=159$ ).

Por meio da análise de regressão linear univariada, apenas as variáveis formação, escola e eficácia coletiva escolar demonstraram relações explicativas consideradas significativas, como pode ser observado na Tabela 1. Isso sugere que essas três variáveis atuam isoladamente e significativamente para explicar a autoeficácia docente. Os resultados expostos na Tabela 1 possibilitam identificar que os participantes desta pesquisa formados em nível de mestrado $(\beta=43,71$; $\mathrm{EP}=13,76 ; \mathrm{p}<0,05)$ e doutorado $(\beta=50,84 ; \mathrm{EP}=22,85 ; \mathrm{p}<0,05)$ percebem-se mais capazes que os demais professores, de modo que a formação explica $9,30 \%$ da variância da autoeficácia docente. Os participantes que lecionam na escola $12(\beta=40,68 ; \mathrm{EP}=17,14 ; \mathrm{p}<0,05)$ foram os que demonstraram maior percepção de autoeficácia docente. A escola em que os professores atuam explica $13,35 \%$ da variância da autoeficácia docente. Entre essas variáveis, a eficácia coletiva escolar foi a que demonstrou maior poder explicativo $(\beta=0,60$; EP $=0,07 ;<0,001)$ sobre a autoeficácia docente, explicando $33,54 \%$ da variância dessa crença.

A seguir realizou-se a análise de regressão linear multivariada, na qual todas as variáveis explicativas, antes verificadas de modo isolado, passaram a ser estudadas de modo conjunto. Na Tabela 2 são apresentadas apenas as variáveis explicativas selecionadas que revelaram relações significativas conjuntas à autoeficácia docente, selecionadas entre todas as variáveis explicativas analisadas na análise de regressão linear univariada (Tabela 1). 
TABELA 1 - ANÁLISE DE REGRESSÃO LINEAR UNIVARIADA PARA ESCORE TOTAL DA ESCALA $\operatorname{AED}(\mathrm{N}=159)$

\begin{tabular}{|c|c|c|c|c|}
\hline Variável & Categorias & Beta (EP)* & Valor-P & $\mathbf{R}^{2}$ \\
\hline AEG & Variável contínua & $0,18(0,10)$ & 0,087 & 0,0220 \\
\hline ECE & Variável contínua & $0,60(0,07)$ & $<0,001$ & 0,3354 \\
\hline Jornada de trabalho & Variável contínua & $0,13(0,08)$ & 0,112 & 0,0165 \\
\hline Jornada cumprida & $\begin{array}{c}\text { Apenas nessa escola (ref.) } \\
\text { Nessa e outra pública } \\
\text { Nessa e outra privada } \\
\text { Nessa e outra pública/privada }\end{array}$ & $\begin{array}{c}-- \\
6,09(9,64) \\
-0,79(11,86) \\
21,62(21,24) \\
\end{array}$ & $\begin{array}{l}0,529 \\
0,947 \\
0,310\end{array}$ & 0,0091 \\
\hline Número de alunos & $\begin{array}{c}30 \text { (ref.) } \\
35 \\
40 \\
45 \\
50 \\
\end{array}$ & $\begin{array}{c}--- \\
1,09(24,03) \\
7,98(23,39) \\
17,89(23,87) \\
57,63(32,19)\end{array}$ & $\begin{array}{l}0,964 \\
0,733 \\
0,455 \\
0,076\end{array}$ & 0,0461 \\
\hline Infraestrutura & $\begin{array}{l}\text { Muito adequada (ref.) } \\
\text { Adequada } \\
\text { Pouco adequada } \\
\text { Insuficiente } \\
\end{array}$ & $\begin{array}{c}-- \\
-10,04(14,03) \\
-16,99(14,32) \\
-21,44(15,69) \\
\end{array}$ & $\begin{array}{l}0,476 \\
0,237 \\
0,174 \\
\end{array}$ & 0,0164 \\
\hline Escola & $\begin{array}{c}1 \text { (ref.) } \\
2 \\
3 \\
4 \\
11 \\
12 \\
13 \\
14 \\
15 \\
16 \\
17 \\
\end{array}$ & $\begin{array}{c}-- \\
13,27(18,07) \\
26,63(14,96) \\
2,97(15,47) \\
-10,76(18,66) \\
40,68(17,14) \\
8,47(15,29) \\
-10,89(20,26) \\
-4,66(15,91) \\
10,09(17,14) \\
-19,93(15,12)\end{array}$ & $\begin{array}{l}0,464 \\
0,077 \\
0,848 \\
0,565 \\
\mathbf{0 , 0 1 9} \\
0,581 \\
0,592 \\
0,770 \\
0,557 \\
0,189 \\
\end{array}$ & 0,1335 \\
\hline Formação & $\begin{array}{l}\text { Licenciatura (ref.) } \\
\text { Pedagogia } \\
\text { Especialização } \\
\text { Mestrado } \\
\text { Doutorado }\end{array}$ & $\begin{array}{c}-- \\
21,78(16,51) \\
10,48(8,54) \\
43,71(13,76) \\
50,84(22,85)\end{array}$ & $\begin{array}{l}0,189 \\
0,222 \\
\mathbf{0 , 0 0 2} \\
\mathbf{0 , 0 2 8}\end{array}$ & 0,0930 \\
\hline $\begin{array}{l}\text { Tempo de atuação na } \\
\text { escola }\end{array}$ & Variável contínua & $0,09(0,08)$ & 0,314 & 0,0067 \\
\hline $\begin{array}{l}\text { Tempo de atuação na } \\
\text { profissão }\end{array}$ & Variável contínua & $0,13(0,08)$ & 0,107 & 0,0170 \\
\hline Idade & Variável contínua & $0,15(0,08)$ & 0,062 & 0,0225 \\
\hline Sexo & $\begin{array}{c}\text { Feminino (ref.) } \\
\text { Masculino }\end{array}$ & $\begin{array}{c}-- \\
5,57(8,46)\end{array}$ & 0,511 & 0,0028 \\
\hline IPVS & Variável contínua & $-0,09(0,09)$ & 0,309 & 0,0066 \\
\hline
\end{tabular}

* Beta: valor da estimativa ou coeficiente angular (slope) na reta de regressão; EP: erro padrão de beta. $\mathrm{R}^{2}$ : coeficiente de determinação (\% de variabilidade da variável resposta explicada pela variável independente). Ref: nível de referência. Variáveis numéricas transformadas em postos (ranks) devido à ausência de distribuição Normal.

FONTE: Casanova (2013). 
Como pode ser observado na Tabela 2, em conjunto, as variáveis formação, eficácia coletiva escolar (ECE) e tempo de atuação na escola evidenciaram relações significativas com a autoeficácia docente. Observando-se a Tabela 2, pode-se verificar que a eficácia coletiva escolar foi a que demonstrou maior poder explicativo ( $\beta=0,69 ; \mathrm{EP}=0,07 ; \mathrm{p}<0,001$ ), explicando $37,90 \%$ da variância da autoeficácia docente. Os professores formados com títulos de especialistas $(\beta=15,85 ; \mathrm{EP}=7,54 ; \mathrm{p}<0,05)$, mestres $(\beta=54,08 ; \mathrm{EP}=13,35 ; \mathrm{p}<0,001) \mathrm{e}$ doutores $(\beta=54,17 ; \mathrm{EP}=19,77 ; \mathrm{p}<0,05)$ perceberam-se mais capazes do que os participantes com titulações menores, destacando-se que os titulados em nível de mestrado demonstraram relação significante com a autoeficácia docente.

TABELA 2 - ANÁLISE DE REGRESSÃO LINEAR MULTIVARIADA PARA ESCORE TOTAL DE ESCALA $\operatorname{AED}(\mathrm{N}=113)$

\begin{tabular}{|c|c|c|c|c|}
\hline Variáveis Selecionadas & Categorias & Beta $($ EP)* & Valor-P & $\mathbf{R}^{2}$ Parcial \\
\hline 1. ECE & Variável contínua & $0,69(0,07)$ & $<0,001$ & 0,3790 \\
\hline \multirow{5}{*}{ 2. Formação } & Licenciatura (ref.) & --- & & \\
\hline & Pedagogia & $22,27(14,44)$ & 0,126 & \\
\hline & Especialização & $15,85(7,54)$ & 0,038 & \\
\hline & Mestrado & $54,08(13,35)$ & $<0,001$ & \\
\hline & Doutorado & $54,17(19,77)$ & 0,007 & 0,1176 \\
\hline 3. Tempo de atuação na escola & Variável contínua & $0,17(0,07)$ & 0,021 & 0,0247 \\
\hline
\end{tabular}

* Beta: valor da estimativa ou coeficiente angular (slope) na reta de regressão; EP: erro padrão de beta. $\mathrm{R}^{2}$ : coeficiente de determinação. Critério Stepwise de seleção de variáveis. $\mathrm{R}^{2}$ Total: 0,5213. Intercepto (EP): 4,25 (9,03); $\mathrm{P}=0,639$. Variáveis numéricas transformadas em postos (ranks) devido à ausência de distribuição Normal.

FONTE: Casanova (2013).

No modelo exposto na Tabela 2, a formação explica 11,76\% da variância da autoeficácia docente. Inserida no modelo conjunto, a variável tempo de atuação na escola pesquisada mostrou-se significativa $(\beta=0,17 ; \mathrm{EP}=0,07 ; \mathrm{p}$ $<0,05$ ), explicando $2,47 \%$ da variância da autoeficácia docente, e não mais a variável escola. Isso parece sugerir que o tempo que um professor atua em determinada unidade escolar é mais importante do que a escola em que ele atua como uma possibilidade explicativa de autoeficácia docente. Essas três variáveis conjuntamente explicam 52,31\% da autoeficácia docente $\left(\mathrm{R}^{2} \mathrm{Total}=0,5231 ; \mathrm{p}\right.$ $>0,05$ ). Analisando-se os resultados expostos na Tabela 2 , pode-se afirmar que os professores com maiores escores de autoeficácia docente foram os com maiores percepções de eficácia coletiva escolar, formados em especialização, mestrado ou doutorado e com maior tempo de atuação na unidade escolar pesquisada. 


\section{Discussão}

Esta pesquisa evidenciou que as crenças de autoeficácia constatadas nesta amostra pareceram ser explicadas pela ação conjunta das variáveis eficácia coletiva escolar, formação e tempo de atuação na escola pesquisada. Os professores aqui participantes percebem-se capazes de atingir os resultados desejados de engajamento e aprendizagem dos alunos, como identificado por meio das medianas observadas. Tais percepções sugerem percepções otimistas e estão em níveis semelhantes de outras pesquisas nacionais, as quais verificaram que os docentes se sentiam moderadamente capazes. (ALVARENGA, 2011; FERREIRA, 2011; IAOCHITE, 2007).

A relação entre autoeficácia docente e formação parece informar que, quanto maior a titulação do professor, maior a percepção de capacidade para utilizar estratégias instrucionais, manejar a sala de aula e estimular o engajamento dos estudantes. Tal constatação coincide com outras pesquisas, tanto internacional (TSCHANNEN-MORAN; JOHNSON, 2011) quanto nacional (ALVARENGA, 2011), e auxilia a destacar a importância da formação do professor, especificamente a formação formal, como um meio de se estimular a percepção de autoeficácia docente. Uma melhor formação, associada a uma maior percepção de autoeficácia docente, pode contribuir indiretamente para a seleção e utilização de estratégias de ensino, para o manejo de sala de aula e para o engajamento dos estudantes. (THOONEN et al., 2011; WOOLFOK-HOY; DAVIS, 2006). Isso ressalta a necessidade de se pensar em uma formação profissional que proporcione condições para o desenvolvimento de habilidades necessárias para a atividade aliada à promoção da crença de autoeficácia para realizar as tarefas inerentes a dada função, dado que uma pessoa pode ter determinada habilidade mas usá-la de modo simples, adequado ou eficiente, dependendo da crença de eficácia que possui em dada circunstância. (BANDURA, 1997). Possivelmente, estimular a participação de professores em programas de pós-graduação contribuirá para se fortalecer as crenças de autoeficácia docente.

Por um lado, a escola em que o professor atua é um aspecto de contexto que demonstrou ser importante para a percepção de autoeficácia docente. Não foram encontradas na literatura analisada informações específicas sobre essa relação, mas autores (AZZI; POLYDORO, 2011; WOOLFOLK-HOY; DAVIS, 2006) têm pontuado que aspectos de contexto, como a infraestrutura da escola, podem se relacionar à autoeficácia docente. (IAOCHITE, 2007; TSCHANNEN-MORAN; JOHNSON, 2011). Por outro lado, os resultados desta pesquisa sugerem que mais importante do que a escola em que o professor atua, é o tempo de atuação 
nesta unidade escolar, pois quanto maior o tempo de atuação em uma mesma escola, maior a percepção de autoeficácia docente. Na literatura analisada, não foi encontrada informação sobre a relação da autoeficácia docente com o tempo de atuação na mesma escola. A literatura mostra que há relação entre a experiência docente e a crença de autoeficácia docente, de modo que professores iniciantes parecem ter crenças de autoeficácia mais frágeis. (FERREIRA, 2011; MACEDO, 2009; TSCHANNEN-MORAN; WOOLFOLK-HOY, 2001; OLIVEIRA; FREITAS, 2010). Porém, pode-se inferir que um professor que atua há mais tempo em uma dada escola não seja um professor iniciante. Tais constatações reforçam o postulado por Paro (2011), destacando a importância de se estruturar condições de trabalho que evitem a rotatividade docente, estimulando o professor a atuar mais tempo em uma mesma escola.

Dentre as variáveis aqui investigadas, a eficácia coletiva escolar demonstrou ser a mais importante para explicar a autoeficácia docente, coincidindo com o verificado por Kurt, Duyar e Çalik (2011). Isso auxilia a destacar a importância da crença de eficácia coletiva escolar, dado que a atuação docente é interdependente da atuação coletiva, como postulado por Bandura (1997). Diante disso, assegurar o término da rotatividade constante, bem como condições de trabalho que privilegiem a dedicação exclusiva à unidade escolar, coloca-se como fator fundamental, pois pode possibilitar condições para a formação coletiva em serviço na própria escola, coerente com a proposta pedagógica dela e necessária para o trabalho pedagógico coletivo. (PARO, 2011; GOMES, 2012). Condições que podem contribuir para o fortalecimento da crença de eficácia coletiva escolar, a qual pode estimular a autoeficácia docente.

As variáveis pessoais, gênero e idade, não se relacionaram com a crença de autoeficácia docente, coincidindo com o verificado em outras culturas. (TSCHANNEN-MORAN; JOHNSON, 2011). Embora a relação da autoeficácia docente com a infraestrutura da escola ora seja significativa (IAOCHITE, 2007) e ora não (GOYA; BZUNECK; GUIMARÃES; 2008), a constatação nesta pesquisa de que o IPVS e a infraestrutura não se relacionam significativamente com a autoeficácia docente parece destacar que as variáveis de contexto, intrinsecamente relacionadas às questões de carreira são mais importantes do que $o$ ambiente físico e social em que a escola está inserida como meios de se fortalecer a crença em questão. Proporcionar boas condições de trabalho, envolvendo os aspectos de valorização de carreira docente, faz-se fundamental para elevar as percepções de autoeficácia docente, pois um professor pode saber como atuar a fim de otimizar as atividades instrucionais que realiza, mas se o contexto em que a atividade docente é realizada não possibilita o pleno exercício de suas capacidades, esse professor pode apresentar um crença de autoeficácia acima de seu desempenho, não por não saber como fazer, mas por se sentir impedido 
de atuar como sabem. (PAJARES, OLAZ, 2008; WOOLFOLK-HOY; DAVIS, 2006). Maior participação de docentes na elaboração de políticas pedagógicas e mais autonomia pedagógica às escolas também podem ser interpretadas como condições de trabalho contextuais, as quais seriam necessárias para o fortalecimento das crenças de autoeficácia docente. (SKAALVIK; SKAALVIK, 2007).

Sabe-se que na prática escolar cotidiana, as variáveis não "atuam" sozinhas, mas sim de modo inter-relacionado. Sem a pretensão de ter considerado todos os aspectos que podem ser relacionar com a autoeficácia docente e a prática cotidiana, entende-se que esta pesquisa possibilitou uma visão integrada sobre vários aspectos pessoais, de atividade docente, contextuais e motivacionais que perpassam o cotidiano escolar, contribuindo para esclarecer alguns aspectos da complexa vivência escolar. No entanto, reconhece-se que a especificidade desta amostra limita a generalização dos conhecimentos aqui apresentados, bem como que os modelos advindos da análise de regressão lineares são provisórios e válidos até que novas evidências possam ser obtidas e ampliar o conhecimento até então alcançado. Apesar dessas limitações, como implicações sugere-se que a formação formal seja elaborada de modo a possibilitar que os docentes possam desenvolver habilidades específicas às suas especialidades conjuntamente às crenças de autoeficácia, como também que a rotatividade docente diminua, de modo a possibilitar que os profissionais passem mais tempo em uma mesma escola, auxiliando a ampliar a crença de eficácia docente.

\section{REFERÊNCIAS}

ALVARENGA, C. E. A. Autoeficácia de professores para utilizarem tecnologias de informática no ensino. 183p. Tese (Doutorado) - Faculdade de Educação, Universidade Estadual de Campinas, Campinas, 2011.

AZZI, R. G.; POLYDORO, S. A. J. O papel da autoeficácia e autorregulação no processo motivacional. In: BORUCHOVITCH, E.; BZUNECK, J. A.; GUIMARÃES, M. S. E. R. Motivação para aprender: aplicações no contexto educativo. Petrópolis: Vozes, 2010. p. 126-144.

AZZI, R. G.; POLYDORO, S. A. J. Contribuições da Teoria Social Cognitiva de Albert Bandura ao Contexto Educativo. In: AZZI, R. G.; GIANFALDONI, M. H. T. A. (Orgs.). Psicologia e Educação. São Paulo: Casa do Psicólogo, 2011. p. 177-198 (Série ABEP Formação, v. 1). 
AZZI, R. G.; POLYDORO, S. A. J.; BZUNECK, J. A. Considerações sobre a auto-eficácia docente. In: AZZI, R. G.; POLYDORO, S. A. J. (Orgs.). Auto-eficácia em diferentes contextos. Campinas-SP: Alínea, 2006. p. 149-159.

BANDURA, A. Exercise of personal and collective efficacy in changing societies. In: BANDURA, A. (Org.). Self-efficacy in changing societies. Cambridge: Cambridge University Press, 1995. p. 1-45.

BANDURA, A. Self-efficacy, the exercise of control. New York: Freeman and Company, 1997.

CASANOVA, D. C. G. Crenças de Eficácia de gestores escolares e de docentes no ensino médio paulista. Tese (Doutorado), Faculdade de Educação, Universidade Estadual de Campinas, Campinas, 2013.

CASTELO, L. B; LUNA, I. N. Crença de autoeficácia e identidade profissional: Estudo com professores do ensino médio. Psicol. Argum., Curitiba, v. 30, n. 68, p. 27-42, jan./ mar. 2012.

CONOVER, W. J.; IMAN, R. I. Rank Transformations as a Bridge Between Parametric and Nonparametric Statistics. The American Statistician, v. 35, n. 3, p. 124-129, 1981.

DUFFY, R. D.; LENT, R. W. Test of a social cognitive model of work satisfaction in teachers. Journal of Vocational Behavior, v. 75, p. 212-223, 2009.

FERREIRA, L. C. M. Relação entre a crença de auto-eficácia docente e a síndrome de burnout em professores do ensino médio. 191 p. Tese (Doutorado) - Faculdade de Educação, Universidade Estadual de Campinas, 2011.

FREITAS, J. A. C.; OLIVEIRA, J. A. Análise da auto-eficácia percebida pelos professores de escolas públicas e privadas de Natal. INTERFACE, Natal, v. 7, n. 2, p. 63-78, jul./dez. 2010.

FUNDAÇÃO SISTEMA ESTADUAL DE ANÁLISE DE DADOS - SEADE. Disponível em: <http://www.seade.gov.br/projetos/ipvs/municipios_pdf.php>. Acesso em: 10 nov. 2012.

GOMES, S. S. Práticas docentes e processos de formação. Educação, Porto Alegre, v. 35, n. 3, p. 414-423, set./dez. 2012.

GOYA, A.; BZUNECK, J. A.; GUIMARÃES, S. E. R. Crenças de eficácia de professores e motivação de adolescentes para aprender Física. Revista Semestral da Associação Brasileira de Psicologia Escolar e Educacional (ABRAPEE), v. 12, n. 1, p. 51-67, 2008.

IAOCHITE, R. T. Auto-eficácia de docentes de educação física. 159p. Tese (Doutorado) - Faculdade de Educação, Universidade Estadual de Campinas, Campinas, 2007.

KURT, T.; DUYAR, I; ÇALIK, T. Are we legitimate yet?: A closer look at the casual relationship mechanisms among principal leadership, teacher self-efficacy and collective efficacy. Journal of Management Development, v. 31, n. 1, p. 71-86, 2011. 
KURZ, T. B.; KNIGHT, S. L. An exploration of the relationship among teacher efficacy, collective teacher efficacy, and goal consensus. Learning Environment Research, v. 7, n. 2, p. 111-128, 2004.

MACEDO, C. I. Crenças de autoeficácia de professores do Ensino Fundamental e suas relações com percepções de apoio na escola. Dissertação (Mestrado em Educação) Universidade Estadual de Londrina, Londrina, 2009.

PAJARES, F; OLAZ, F. Teoria social cognitiva e autoeficácia: uma visão geral. In: BANDURA, A; AZZI, R. G.; POLYDORO, S. (Orgs.). Teoria Social Cognitiva. Tradução de Ronaldo Cataldo Costa. Porto Alegre: Artmed, 2008. p. 97-114.

PARO, V. H. Crítica da estrutura da escola. São Paulo: Cortez, 2011. 248 p.

RODRÍGUEZ, S.; NÚÑEZ, J. C.; VALLE, A.; BLAS, R.; ROSARIO, P. Auto-eficacia docente, Motivación del Profesor y Estrategias de enseñanza. Escritos de Psicología, v. 3, n. 1, p. 1-7, 2009.

SALANOVA, M.; LLORENS, S. Yes, I Can, I Feel Good, and I Just Do It! On Gain Cycles and Spirals of Efficacy Beliefs, Affect, and Engagement. Applied Psychology: An International Review, v. 60, n. 2, p. 255-285, 2011.

SKAALVIK, E. M.; SKAALVIK, S. Dimension softeacher self-efficacy and relations with strain factors, perceived collective teacher efficacy, and teacher burnout. Journal of Educational Psychology, v. 99, p. 611-625, 2007.

THOONEN, E. E. J.; SLEEGERS, P. J. C.; PEETSMA, T. T. D.; OORT, F. J. Can Teachers Motivate Students to Learn? Educational Studies, v. 37, n. 3, p. 345-360, 2011.

TSCHANNEN-MORAN, M.; BARR, M. Fostering student achievement: The relationship between collective teacher efficacy and student achievement. Leadership and Policy in Schools, v. 3, p. 187-207, 2004.

TSCHANNEN-MORAN, M.; GAREIS, C. Principals' sense of efficacy: Assessing a promising construct. Journal of Educational Administration, v. 42, p. 573-585, 2004.

TSCHANNEN-MORAN, M.; JOHNSON, D. Exploring Literacy Teachers' Self-Efficacy Beliefs: Potential Sources at Play. Teaching and Teacher Education: An International Journal of Research and Studies, v. 27, n. 4, p. 751-761, 2011.

TSCHANNEN-MORAN, M.; WOOLFOLK- HOY, A. Teacher efficacy: Capturing and elusive construct. Teaching and Teacher Education, v. 17, p. 783-805, 2001.

Texto recebido em 24 de setembro de 2015 .

Texto aprovado em 10 de novembro de 2015. 